\title{
CYCLIC GROUP ACTIONS ON RIEMANN SURFACES
}

\author{
MAHGOUB YAHIA
}

\begin{abstract}
We study the actions of $\mathbf{Z} / m$, the cyclic group of order $m$, on Riemann surfaces which generate the bordism group $\mathscr{U}_{2}^{\mathbf{Z} / m}$. We analyse $\mathscr{U}_{2}^{\mathbf{Z} / m}$ by means of fixed point structure using the technique of "bordism with families of slice types" [4].
\end{abstract}

1. Introduction. Although smooth actions of cyclic groups on closed unitary or oriented surfaces can be easily classified up to equivariant diffeomorphism, there are contexts in which one needs a more precise understanding of such actions. For example, one natural question concerns the $G$-signature which can be realized by oriented $G$-surfaces. We shall give a family of $\mathbf{Z} / m$ surfaces whose $G$-signatures generate the imaginary summand of the localized representation $\operatorname{ring} R(\mathbf{Z} / m) \otimes \mathbf{Z}\left[\frac{1}{2}\right]$. The existence of such families has proved to be important in some contexts (compare [6].)

The author would like to express his thanks to C. Kosniowski for his help.

2. The generators. In this paper the generators fall into two classes- "primitive" and "induced". The primitive generators are of three types. The first type needs no further comment.

2.1. $C P^{1}$ : the complex projective line $C P^{1}$ with trivial $\mathbf{Z} / m$ action.

In order to describe the second type we shall first give some notation.

Let $V_{j}$ be the complex line $C$ with $\mathbf{Z} / m$ action given by multiplication by $\xi^{j}=\exp (2 \pi i j / m)$. Then the one-dimensional irreducible complex $\mathbf{Z} / m$-modules are $V_{j}, j=1,2, \ldots, m$. The second family is then given as follows:

2.2. $C P\left(C \oplus V_{j}\right), j=1,2, \ldots,[(m-1) / 2]$ : the complex projective line with $\mathbf{Z} / m$ action given by $\left[z_{0}, z_{1}\right] \rightarrow\left[z_{0}, \xi^{j} z_{1}\right]$.

The third type requires some explanation. We begin with a list of formal names for the examples.

2.3. $S(m, n, j, h), h|n| m$, where $(j, n / h)=1$ and $1 \leqslant j \leqslant((n / h)-1) / 2$.

The following discussion gives the construction for such examples.

For given $h$ and $j$ let $q$ be the unique integer satisfying

(i) $(q, n)=h$,

(ii) $j q / h=-1 \bmod (n / h)$,

(iii) $1<q \leqslant n-1$.

Received by the editors July 13, 1981 and, in revised form, August 29, 1983.

1980 Mathematics Subject Classification. Primary 57S17, 57R85; Secondary 30F20, 57R50.

(C1984 American Mathematical Society $0002-9939 / 84 \$ 1.00+\$ .25$ per page 
Let $A(k), k=1,2, \ldots, q$, be $q$ (two-dimensional) discs in $C \times \mathbf{R}$, i.e. $A(k)=$ $\left\{(z, k) C \times \mathbf{R}:|z|^{2} \leqslant 1\right\}$. Also let $A(k, j), 0 \leqslant j \leqslant 4 n-1$, be $4 n$ points on the boundary of the disc $A(k)$ defined by

$$
A(k, j)=(\exp (\pi i j / 2 n), k) \in C \times \mathbf{R} .
$$

Take $n(q-1)$ rectangular shaped strips and label their vertices in the following way:

$$
\begin{aligned}
& \begin{array}{c}
B(2 r+3,4 s+3) \\
B(2 r+2,4 s+1)
\end{array} \quad \begin{array}{r}
B(2 r+3,4 s+2) \\
r=0,1, \ldots,[(q-2) / 2] \\
s=0,1, \ldots, n-1 \\
B(2 r+2,4 s+3)
\end{array} \\
& B(2 r+2,4 s+1) \square\left[\begin{array}{rl}
B(2 r+2,4 s) \\
r=0,1, \ldots,[(q-2) / 2] \\
s=0,1, \ldots, n-1
\end{array}\right.
\end{aligned}
$$

Embed these strips into $C \times \mathbf{R}$ so that the edges $(B(k, j), B(k, j+1))$ and $(A(k, j), A(k, j+1))$ are identified whenever the edge $(B(k, j), B(k, j+1))$ is defined. The result is a unitary surface $M^{1}$ with boundary. (See Figure 1, for the case $m=4, j=1, q=2=h$ and Figure 2 for the case $m=6, j=1, h=3$ and $q=3$.)

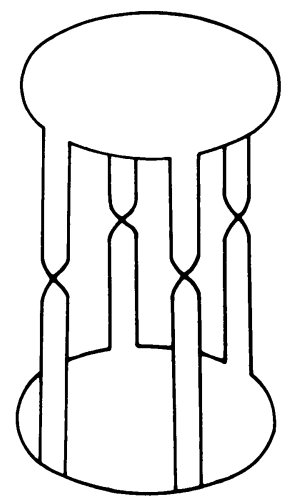

FIGURE 1

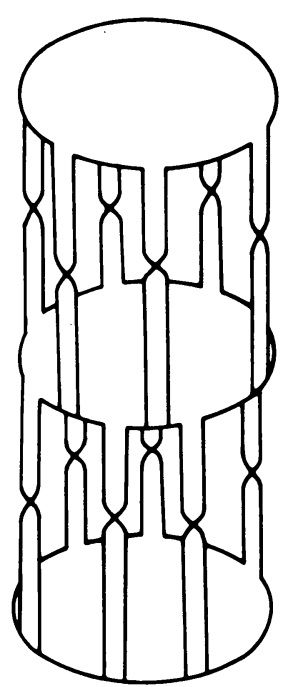

FIGURE 2

There is an action of $\mathbf{Z} / m$ on $C \times \mathbf{R}$ given by $(z, t) \rightarrow\left(\xi^{m / n} z, t\right)$ and if the rectangular strips are embedded symmetrically, then this action of $\mathbf{Z} / m$ on $C \times \mathbf{R}$ induces an action of $\mathbf{Z} / m$ on $M^{1}$. The resulting action on $M^{1}$ induces an action on the boundary of $M^{1}$. It is easy to see that the boundary of $M^{1}$ consists of $h$ circles. 
The $\mathbf{Z} / m$ action on $h$ circles can be extended uniquely to an action on $h$ discs. By attaching such $h$ discs to $M^{1}$ and smoothing out the corners we get a closed unitary surface of genus $(q-1)[(n-1) / 2]$ with a $\mathbf{Z} / m$ action.

Finally we come to the "induced" examples:

2.4. $\mathbf{Z} / m \times_{\mathbf{z} / k} M$ where $k \mid m$ and $M$ is an example from (2.1)-(2.3).

2.5 TheOREM. Let $G=\mathbf{Z} / m$ and $Q=\{n: n \mid m, 1<n \leqslant m\}$. Then $\mathscr{U}_{2}^{G}$ is freely generated by surfaces $\mathbf{Z} / m \times_{\mathbf{Z} / n} M$ where $n \in Q$ and $M$ is a surface from the following list:

$$
\begin{aligned}
& C P^{1}, \\
& C P\left(C \oplus V_{j}\right) \quad \text { where } j=1,2, \ldots, n / 2, \\
& S(n, n / t, j / t, 1) \quad \text { where } 1<j<n / 2 \text { and }(n, j)=t \neq j, \\
& S\left(n, p^{r}, p^{r-1}-1, p\right) \quad \text { where } n / j=p^{r}, r \neq 1, \\
& S(n, p, 1,1) \quad \text { where } n / j=p \neq 2, \\
& k_{1} S\left(n, n / j, p_{j} d-1, p_{1}\right)+k_{2} S\left(n, n / j, p_{1} d-1, p_{2}\right) \\
& \quad \text { where } n / j=p_{1} p_{2} d, d \geqslant 1 \text { and } p_{1} \neq p_{2} \text { are primes such that } \\
& k_{1} p_{1}+k_{2} p_{2}=1 .
\end{aligned}
$$

From the last example in 2.5 we have the following result (cf. [3, (9)]).

2.6 COROLLARY. There exist a closed unitary 2-manifold $M$ and $a \mathrm{Z} / m$ action on $M$ with precisely one fixed point if $m$ is not a power of a prime.

2.7. Slice types (cf. [4]). We shall study $\mathscr{U}_{2}^{G}$ using the "slice type" exact sequences of [4, Chapter I]. Abstractly, a slice type for $G$ is a pair $[H, U]$ with $H$ a closed subgroup of $G$ and $U$ an $H$-representation with $U^{H}=\{0\}$. If $M$ is a smooth $G$-manifold, then by [4, p. 7] we have a slice type $\left[H_{x} ; U_{x}\right]$ at each $x \in M$. Given a suitable family $F$ of slice types and a subfamily $F^{\prime}$, one can define bordism groups $\mathscr{U}_{*}\left(F, F^{\prime}\right)$. These fit into several exact sequences of a familiar nature (e.g., [4, 1.3.2, p. 11]). If $F$ is obtained from $F^{\prime}$ by adding a single slice type [ $\left.H ; U\right]$, then there is a natural isomorphism from $\mathscr{U}_{*}\left(F, F^{\prime}\right)$ to a group $\mathscr{U}_{*}[H, U]$ of bordism classes of $G$-vector bundles $[4,1.4 .4$, p. 16]. Specifically, one considers bundles $E \downarrow N$ such that $N$ is the set of points with slice type $[H, U]$. The isomorphism is given by taking the equivariant normal bundle to a component of the fixed point set of $H$ [4, Corollary 1.5 .3 , p. 19].

These results give us an inductive method for computing $\mathscr{U}_{*}^{G}[F]$.

3. Proof of Theorem 2.5. The $Z / m$ slice types are:

$$
\begin{aligned}
& \sigma_{1,0}=[1 ; 0], \\
& \sigma_{n, 0}=[\mathbf{Z} / n ; 0], \quad n \mid m, \\
& \sigma_{n, j}=\left[\mathbf{Z} / n ; V_{j}\right], \quad j=1,2, \ldots, n-1 \text { and } n \mid m .
\end{aligned}
$$

Order the slice types as follows:

(i) $\sigma_{n, 0}<\sigma_{l, j}$, if $j=1,2, \ldots, l-1$,

(ii) $\sigma_{n, i}<\sigma_{l, j}$, if $n<l$, 
(iii) $\sigma_{n, i}<\sigma_{n, j}$, if $i<j$,

i.e., we order first by dimension, then if the dimensions are equal we use the order of the isotropy subgroups $\mathbf{Z} / n<\mathbf{Z} / l$ for $n<l$. If these are also equal we order lexicographically using $V_{i}<V_{j}$ for $i<j$.

Define sets $F_{n, j}$ by $F_{n, j}=\left\{\rho: \rho \leqslant \sigma_{n, j}\right\}$. It is immediate that $F_{n, j}$ is a family of slice types so we have the exact sequence for the pair $\bar{F}_{n, j} \subseteq F_{n, j}$ where

$$
\begin{aligned}
& \bar{F}_{n, j}=F_{n, j}-\left\{\sigma_{n, j}\right\} \cdots \rightarrow \mathscr{U}_{3}^{\mathbf{Z} / m}\left[\sigma_{n, j}\right] \stackrel{\partial_{n j}^{3}}{\rightarrow} \mathscr{U}_{2}^{\mathbf{Z} / m}\left[\bar{F}_{n, j}\right] \\
& \rightarrow \mathscr{U}_{2}^{\mathbf{Z} / m}\left[F_{n, j}\right] \stackrel{\nu}{\rightarrow} \mathscr{U}_{2}^{\mathbf{Z} / m}\left[\sigma_{n, j}\right] \stackrel{\partial}{\rightarrow} \mathscr{U}_{1}^{\mathbf{Z} / m}\left[\bar{F}_{n, j}\right] \rightarrow \cdots .
\end{aligned}
$$

From [4, (1.5), (1.6) and (3.2)] we have

$$
\mathscr{U}_{3}^{\mathbf{Z} / m}\left[\sigma_{n, j}\right] \cong \mathscr{U}_{3}\left(B\left(\mathbf{Z} / m \times_{\mathbf{Z} / n} U(1)\right)\right) .
$$

We see that $\mathscr{U}_{3}^{\mathbf{Z} / m}\left[\boldsymbol{\sigma}_{n, j}\right]$ is generated freely as a $\mathbf{Z}$-module by

$$
S\left(V_{m}\right) \times_{\mathbf{z} / n} V_{j}=S\left(V_{n / m}\right) \times V_{j} .
$$

Similarly $\mathscr{U}_{2}^{\mathbf{Z} / m}\left[\sigma_{n, j}\right]$ is freely generated by $\mathbf{Z} / m \times_{\mathbf{Z} / n} V_{j}$ while $\mathscr{U}_{2}^{\mathbf{Z} / m}\left[\sigma_{n, 0}\right]$ is generated by $\mathbf{Z} /(m / n) \times C P$.

It is clear that $\operatorname{Im} \partial_{n, j}^{3}=0$ so that the sequence reduces to

$$
0 \rightarrow \mathscr{U}_{2}^{\mathbf{Z} / m}\left[\bar{F}_{n, j}\right] \rightarrow \mathscr{U}_{2}^{\mathbf{Z} / m}\left[F_{n, j}\right] \rightarrow \operatorname{ker} \partial_{n, j} \rightarrow 0,
$$

which splits since ker $\partial_{n, j}$ is free. Therefore

$$
\mathscr{U}_{2}^{\mathbf{Z} / m}\left[F_{n, j}\right] \cong \mathscr{U}_{2}^{\mathbf{Z} / m}\left[\bar{F}_{n, j}\right] \oplus \operatorname{ker} \partial_{n, j} .
$$

Hence

$$
\mathscr{U}_{2}^{\mathbf{Z} / m}\left[F_{n, j}\right] \cong \underset{\substack{k, j \\ 1 \leqslant k \leqslant n \\ 1 \leqslant j \leqslant k-1}}{\bigoplus} \operatorname{ker} \partial_{k, j}
$$

We also have

$$
\begin{gathered}
\mathscr{U}_{2}^{\mathbf{Z} / m}\left[\sigma_{n, j}\right] \cong \mathbf{Z} \cdot(\mathbf{Z} / m \times \mathbf{Z} / n \\
\left.\mathscr{U}_{2}\right), \quad j=1,2, \ldots, n-1, \\
\left.\mathbf{Z} / \sigma_{n, 0}\right] \cong \mathbf{Z} \cdot(\mathbf{Z} /(m / n) \times C P) .
\end{gathered}
$$

3.1 Lemma.

$$
\mathscr{U}_{2}^{\mathbf{Z} / m}=\bigoplus_{n \mid m} \mathbf{Z} \cdot(\mathbf{Z} /(m / n) \times C P) \oplus \bigoplus_{\substack{n, j \\ 1 \leq n \leq m \\ 1 \leqslant j \leqslant n-1}} \mathbf{Z} \cdot\left(\alpha\left(\mathbf{Z} / m \times \times_{\mathbf{Z} / n} V_{j}\right)\right),
$$

where

(a) $\alpha=1$ if $n / j$ is not a power of a prime,

(b) $\alpha=p$ if $n / j=p^{r}$ for some prime $p$ and a nonnegative integer $r$.

PROof. The proof consists of Lemmas 3.3 and 3.4 given below. The following will enable us to calculate $\nu(M)$ where $M$ comes from 2.1-2.4. 


\subsection{LEMMA.}

$$
\begin{aligned}
& \nu\left(C P^{1}\right)=C P^{1}, \\
& \nu\left(C P\left(C \oplus V_{j}\right)\right)=V_{j}+V_{m-j}, \\
& \nu(S(m, n, j, h))=q V_{m / n}+\mathbf{Z} / m \times_{\mathbf{Z} /(m / h)} V_{j(m / n)}, \quad h \neq 1, \\
& \nu(S(m, n, j, 1))=V_{j(m / n)}+q V_{m / n},
\end{aligned}
$$

where $\nu=\sum_{k, j} \nu_{k, j}$, and $\nu_{k, j}(M)$ is the normal bundle (to the set of points fixed by $\mathbf{Z} / k$ ) of type $\sigma_{k, j}$.

ProOF. Follows directly from the definition of the surfaces.

The next four results are the main steps needed to prove Theorem 2.5.

3.3 LEMMA. From the examples in 2.5 we can construct two-dimensional unitary $\mathbf{Z} / m$ manifolds $M_{j}$ such that $\nu\left(M_{j}\right)=V_{j}+$ lower order, where

(a) $\alpha=1$ if

(i) $j>m / 2$,

(ii) $1<j<m / 2$ and $j \neq(m, j)$,

(iii) $1 \leqslant j<m / 2, j \mid m$ and $m / j=p_{1} p_{2} d$ where $p_{1} \neq p_{2}$ are primes and $d \geqslant 1$.

(b) $\alpha=p$ if $1 \leqslant j \leqslant m / 2, j \mid m$ and $m / j=p^{r}$ for some prime $p$ and a positive integer $r$.

Proof. (a) (i) $j>m / 2$, let $M_{j}=C P\left(C \oplus V_{j}\right)$.

(ii) $1<j<m / 2$ and $j \neq(m, j)$.

Let $(m, j)=t$; then $(m / t, j / t)=1$. Set $M_{j}=S(m, m / t, j / t, 1)$. By $3.2 \nu\left(M_{j}\right)=V_{j}$ $+q V_{t}$.

(iii) $1 \leqslant j<m / 2, j \mid m$ and $m / j=p_{1} p_{2} d$ where $p_{1} \neq p_{2}$ are primes and $d \geqslant 1$.

Set $N_{1}=S\left(m, m / j, p_{2} d-1, p_{1}\right)$, and $N_{2}=S\left(m, m / j, p_{1} d-1, p_{2}\right)$. Then

$$
\nu\left(N_{1}\right)=p_{1} V_{j}+\mathbf{Z} / m \times \mathbf{Z}_{/ p_{2} d j} V_{\left(p_{2} d-1\right) j},
$$

and

$$
\nu\left(N_{2}\right)=p_{2} V_{j}+\mathbf{Z} / m \times \times_{\mathbf{Z} / p_{1} d j} V\left(p_{1} d-1\right) j .
$$

Since $p_{1} \neq p_{2}$ are primes we can construct $M_{j}$ such that $\nu\left(M_{j}\right)=V_{j}+$ lower order.

(b) If $1 \leqslant j \leqslant m / 2, j \mid m$ and $m / j=p^{r}$ for $p$ prime and $r>0$, set

$$
\begin{aligned}
& M_{j}=S\left(m, p^{r}, p^{r-1}-1, p\right) \text { for } r \neq 1, \\
& M_{j}=S(m, p, 1,1) \text { for } r=1, p \neq 2, \\
& M_{j}=C P\left(C \oplus V_{j}\right) \text { for } r=1 \text { and } p=2 .
\end{aligned}
$$

For the case $r \neq 1$, we have

$$
\nu\left(M_{j}\right)=p V_{j}+\mathbf{Z} / m \times \times_{\mathbf{Z} / j p^{r-1}} V_{j\left(p^{r-1}-1\right)} .
$$

For the case $r=1$, we have $\nu\left(M_{j}\right)=p V_{j}$. 
3.4 LEMMA. Suppose $M$ is a two-dimensional unitary $\mathbf{Z} / m$ manifold whose fixed point set has normal bundle $\nu(M)=a_{0} V_{j}+$ lower order where $j \mid m$ and $m / j=p^{r}$ for some prime $p$. Then $p \mid a_{0}$.

Proof. In view of Lemma 3.3, without loss of generality we may assume

$$
\nu_{\mathbf{Z} / m}(M)=a_{0} V_{j}+a_{1} V_{j(i)}+\cdots+a_{k-1} V_{j(k-1)}+a_{k} V_{j(k)}
$$

where $m / j(i)=p^{r+1}$ and $(j(k), p)=1$. Note that $j(i)=p j(i+1)$. Restricting the $\mathbf{Z} / m$ action to a $\mathbf{Z} / j(k-1)$ action, we see that

$$
\nu_{\mathbf{Z} / j(k-1)}\left(R_{j(k-1)}\left(M_{j}\right)\right)=a_{k} V_{j(k)}+N_{k}^{\prime} .
$$

Here $R_{j(k-1)}$ is $M_{j}$ with the $\mathbf{Z} / j(k-1)$ action given by the inclusion $\mathbf{Z} / j(k-1) \subseteq$ $\mathbf{Z} / m$ and $N_{k}^{\prime}$ is a manifold with trivial $\mathbf{Z} / j(k-1)$ action. By [2, (4)] $p \mid a_{k}$. By the previous lemma we can construct a manifold $N_{j(k)}$ such that $\nu\left(N_{j(k)}\right)=a_{k} V_{j(k)}+$ lower isotropy, and $M_{j}-N_{j(k)}$ has a $\mathbf{Z} / m$ action. Restrict to the associated $\mathbf{Z} / j(k-2)$ action; then

$$
\nu_{\mathbf{Z} / j(k-2)}\left(R_{j(k-2)}\left(M_{j}-N_{j(k)}\right)\right)=a_{k-1} V_{j(k-1)}+N_{k-1}
$$

where $N_{k-1}$ is a manifold with trivial $\mathbf{Z} / j(k-2)$ action. Again by [2, (4)] we have $p \mid a_{k-1}$. Now we can construct a manifold $N_{j(k-1)}$ such that $\nu\left(N_{j(k-1)}\right)=a_{j-1} V_{j(k-1)}$ + lower isotropy by the previous lemma. Repeating this process we see that $p \mid a_{i}$, $i=0,1, \ldots, k$. In particular, $p \mid a_{0}$.

3.5 LeMMA (CF. [3, (7.7)]). Suppose $\mathbf{Z} / m$ is the cyclic group of order $m, \mathbf{Z} / n$ is a subgroup of $\mathbf{Z} / m$, and $\bar{F}=F$ are families of slice types of $\mathbf{Z} / n$. If $F-\bar{F}=\left\{\sigma_{s, j}\right\}$, then the extension map

$$
E_{m, n}: \mathscr{U}_{i}^{\mathbf{Z} / n}[F, \bar{F}] \rightarrow \mathscr{U}_{i}^{\mathbf{Z} / m}[F, \bar{F}],
$$

defined by $M \rightarrow \mathbf{Z} / m \times_{\mathbf{Z} / n} M$, is an isomorphism for $i=2$ and a monomorphism for $i=1$.

Proof. Let $i=2$, then $\mathscr{U}_{2}^{\mathbf{Z} / n}[F, \bar{F}] \cong \mathscr{U}_{2}^{\mathbf{Z} / n}\left[\sigma_{s, j}\right]$ is freely generated as a $\mathbf{Z}$ module by $\mathbf{Z} / n \times{ }_{\mathbf{Z} / s} V_{j}$ for $j \geqslant 1$, and by $\mathbf{Z} / n \times{ }_{\mathbf{Z} / s} C P^{1}$ for $j=0$.

Similarly $\mathscr{U}_{2}^{\mathbf{Z} / m}[F, \bar{F}] \cong \mathscr{U}_{2}^{\mathbf{Z} / m}\left[\sigma_{s, j}\right]$ is freely generated by $\mathbf{Z} / m \times{ }_{\mathbf{Z} / s} V_{j}$ for $j \geqslant 1$ and by $\mathbf{Z} / m \times{ }_{\mathbf{Z} / s} C P^{1}$ for $j=0$. The result follows since $E_{m, n}$ takes generators onto generators.

Now let $i=1$; then

$$
\mathscr{U}_{1}^{\mathbf{Z} / n}[F, \bar{F}] \cong \mathscr{U}_{1}^{\mathbf{Z} / n}\left[\sigma_{s, j}\right]=0, \text { for } s \geqslant 2 .
$$

Similarly

$$
\mathscr{U}_{1}^{\mathbf{Z} / m}[F, \bar{F}] \cong 0, \text { for } s \geqslant 2 .
$$

If $s=1$, then $\sigma_{s, j}=[1 ; 0]$, and

$$
\mathscr{U}_{1}^{\mathbf{Z} / n}[F, \bar{F}]=\mathscr{U}_{1}^{\mathbf{Z} / n}[1 ; 0], \quad \mathscr{U}_{1}^{\mathbf{Z} / m}[F, \bar{F}]=\mathscr{U}_{1}^{\mathbf{Z} / m}[1 ; 0] .
$$

By [2, (4)] $\mathscr{U}_{1}^{\mathrm{Z} / n}[1 ; 0]$ is generated by $S\left(U_{1}\right)$, where $U_{1}$ is $C$ with $\mathrm{Z} / n$ acting by multiplication by $\exp (2 \pi i / n)$, and $\mathscr{U}_{1}^{\mathbf{Z} / m}[1 ; 0]$ is generated by $S\left(V_{1}\right)$, where $V_{1}$ is $C$ 
with $\mathbf{Z} / m$ acting by multiplication by $\exp (2 \pi i / m)$. Consider

$$
E_{m, n}: \mathscr{U}_{1}^{\mathbf{Z} / n}[1 ; 0] \rightarrow \mathscr{U}_{1}^{\mathbf{Z} / m}[1 ; 0] \quad \text { where } S\left(U_{1}\right) \rightarrow \mathbf{Z} / m \times_{\mathbf{Z} / n} S\left(U_{1}\right) .
$$

We can construct a manifold $M=S(m, m, 1, m / n)$ so that

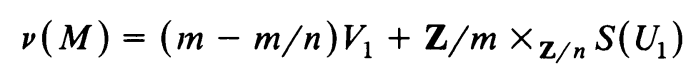

by 3.2. Therefore

$$
-(m-(m / n)) S\left(V_{1}\right)=\mathbf{Z} / m \times_{\mathbf{Z} / n} S\left(U_{1}\right) \text { in } \mathscr{U}_{1}^{\mathbf{Z} / m}[1 ; 0] .
$$

Since $S\left(V_{1}\right)$ has order $m$, we have

$$
\mathbf{Z} / m \times_{\mathbf{Z} / n} S\left(U_{1}\right)=(m / n) S\left(V_{1}\right) \text { in } \mathscr{U}_{1}^{\mathbf{Z} / m}[1 ; 0],
$$

i.e.,

$$
E_{m, n}\left(S\left(U_{1}\right)\right)=(m / n) S\left(V_{1}\right) .
$$

But $S\left(U_{1}\right)$ is of order $n$, so it follows that $E_{m, n}$ is a monomorphism for $i=1$.

3.6 EXTENSION Lemma (Compare With [3, (7.8)]). Suppose $\mathbf{Z} / m$ is a cyclic group of order $m$ and $\mathbf{Z} / n$ is a subgroup of $\mathbf{Z} / m$ and $\bar{F} \subseteq F$ are families of slice types of $\mathbf{Z} / n$. Then $E_{m, n}: \mathscr{U}_{2}^{\mathbf{Z} / n}[F, \bar{F}] \rightarrow \mathscr{U}_{2}^{\mathbf{Z} / m}[F, \bar{F}]$ is an isomorphism.

PROoF. The families of slice types of $\mathbf{Z} / n$ can be written in increasing order as $\phi=F_{-1} \subseteq F_{0} \subseteq F_{1} \subseteq \cdots$ where $F_{i}-F_{i-1}=\left\{\sigma_{s, j}\right\} ; \sigma_{s, j}$ a slice type of $\mathbf{Z} / n$. By induction on $i-j$ we prove that

$$
E_{m, n}: \mathscr{U}_{2}^{\mathbf{Z} / n}\left[F_{i}, F_{j}\right] \rightarrow \mathscr{U}_{2}^{\mathbf{Z} / m}\left[F_{i}, F_{j}\right], \quad i>j,
$$

is an isomorphism. For $i-j=1$ this is immediate from 3.5.

Suppose the statement is true for all $i$ and $j$ with $i-j<k$, where $k \geqslant 2$. Consider $i-j=k$. There is then the triple $F_{i} \supseteq F_{i-1} \supseteq F_{j}$ and the commutative diagram

$$
\begin{aligned}
& \cdots \rightarrow \mathscr{U}_{3}^{\mathbf{Z} / n}\left[\boldsymbol{\sigma}_{s, j}\right] \rightarrow \mathscr{U}_{2}^{\mathbf{Z} / n}\left[F_{i-1}, F_{j}\right] \rightarrow \mathscr{U}_{2}^{\mathbf{Z} / n}\left[F_{i}, F_{j}\right] \rightarrow \mathscr{U}_{2}^{\mathbf{Z} / n}\left[\sigma_{s, j}\right] \rightarrow \mathscr{U}_{1}^{\mathbf{Z} / n}\left[F_{i-1}, F_{j}\right] \rightarrow \cdots \\
& \downarrow E^{1} \quad \downarrow E^{2} \quad \downarrow E^{3} \quad \downarrow E^{4} \quad \downarrow E^{5} \\
& \cdots \rightarrow \mathscr{U}_{3}^{\mathbf{Z} / m}\left[\sigma_{s, j}\right] \rightarrow \mathscr{U}_{2}^{\mathbf{Z} / m}\left[F_{i-1}, F_{j}\right] \rightarrow \mathscr{U}_{2}^{\mathbf{Z} / m}\left[F_{i}, F_{j}\right] \rightarrow \mathscr{U}_{2}^{\mathbf{Z} / m}\left[\sigma_{s, j}\right] \rightarrow \mathscr{U}_{1}^{\mathbf{Z} / m}\left[F_{i-1}, F_{j}\right] \rightarrow \cdots
\end{aligned}
$$

By 3.5 , induction and the five-lemma $E^{3}$ is an epimorphism. Consider

$$
\begin{aligned}
& \text { ว: } \mathscr{U}_{3}^{\mathbf{Z} / m}\left[\sigma_{s, j}\right] \rightarrow \mathscr{U}_{2}^{\mathbf{Z} / m}\left[F_{i-1}, F_{j}\right], \\
& E=S\left(V_{m}\right) \times_{\mathbf{z} / s} V_{j} \rightarrow S\left(V_{m}\right) \times_{\mathbf{z} / s} S\left(V_{j}\right) .
\end{aligned}
$$

Clearly $\partial E$ is bounded by $D\left(V_{m}\right) \times_{\mathrm{z} / s} S\left(V_{j}\right)$. Therefore $\partial$ is the zero homomorphism. Again by induction and the five-lemma $E^{3}$ is a monomorphism and hence an isomorphism.

Lemma 3.1 then follows from 3.3 and 3.4. Theorem 2.5 follows from 3.1, 3.3 and the extension lemma.

4. The $G$-signature (cf. [6]). In this section we show that the $G$-signatures of these examples generate the imaginary summand of $R(\mathbf{Z} / m) \otimes \mathbf{Z}\left[\frac{1}{2}\right]$ when $m$ is odd. 
Let $\Omega_{*}^{\mathbf{Z} / m}$ be the equivariant oriented bordism group. Consider the natural signature homomorphism

$$
\text { sign: } \Omega_{2}^{\mathbf{Z} / m} \otimes \mathbf{Z}\left[\frac{1}{2}\right] \rightarrow R(\mathbf{Z} / m) \otimes \mathbf{Z}\left[\frac{1}{2}\right] .
$$

Let

$$
\tilde{R}(\mathbf{Z} / m)=\operatorname{coker}(R(1) \rightarrow R(\mathbf{Z} / m))=\mathbf{Z}[x] /\left\langle 1+x+\cdots+x^{m-1}\right\rangle
$$

(tensored with $\left.\mathbf{Z}\left[\frac{1}{2}\right]\right)$. In $R(\mathbf{Z} / m) x-1$ is invertible with inverse

$$
m^{-1}\left(1+2 x+\cdots+m x^{m-1}\right) .
$$

From [1, (1.1.5)] we have the character formula

$$
\operatorname{Sign}_{\mathbf{z} / m}(S)(g)=e\left(\nu\left(S^{g}: S\right)\right)^{-1}(g) \operatorname{Sign}\left(S^{g}\right) \in C
$$

(where $e(E)$ is the Euler class of $E$ ), valid when $\nu\left(S^{g}: S\right)$ is $G$-trivial and, hence, we have the following formula from Lemma 3.2.

$$
\operatorname{Sign}_{\mathbf{Z} / m}(S(m, m, j, h))=q \frac{x+1}{x-1}+\frac{x^{m}-1}{x^{m / h}-1} \frac{x^{j}+1}{x^{j}-1} .
$$

Let $\alpha_{j}=\left(x^{j}+1\right) /\left(x^{j}-1\right)$ and $(j, m)=1$. Then

$$
\operatorname{Sign}_{\mathbf{z} / m}(S(m, m, j, h))=2 \alpha_{1}+\alpha_{j}
$$

when $2 j=-1 \bmod m$, hence

$$
2 \alpha_{2 j}+\alpha_{-j}=\alpha_{j}^{-1} \in \operatorname{Im} \operatorname{Sign}_{\mathbf{z} / m} .
$$

\section{REFERENCES}

1. M. F. Atiyah and G. B. Segal, The index of elliptic operators. II, Ann. of Math. (2) 87 (1968), 485-530.

2. P. E. Conner and E. E. Floyd, Periodic maps which preserve a complex structure, Bull. Amer. Math. Soc. 70 (1964), 574-579.

3. __ Maps of odd period, Ann. of Math. (2) 84 (1966), 132-256.

4. C. Kosniowski, Actions of finite abelian groups, Pitman, New York, 1978.

5. $\ldots$, Generators of Z/p bordism ring, Math. Z. 149 (1976), 121-130.

6. I. Madsen and M. Rothenberg, On the classification of $G$-spheres (preprint).

School of Mathematical Sciences, University of Khartoum, P.O. Box 321, Khartoum, Sudan 